\title{
Viewpoint on handling anti-TNF failure in psoriasis
}

\author{
Stefanie Bracke $\cdot$ Jo Lambert
}

Received: 17 July 2013/Revised: 19 September 2013/Accepted: 20 September 2013

(C) Springer-Verlag Berlin Heidelberg 2013

\begin{abstract}
An association among the occurrence of antidrug antibodies (ADAs), diminished trough serum drug levels (TSDLs) and non-response or loss of response has been described for several tumor necrosis factor alpha (TNF) blocking agents in a variety of diseases, including psoriasis. In a series of ten psoriasis patients with primary or secondary failure, or adverse reactions during anti-TNF therapy, we measured ADAs and TSDLs in patient serum using radioimmunoassay and ELISA, respectively. By proposing a treatment algorithm derived from research in this field, we show that measuring ADAs and TSDLs in psoriasis patients provides a more structured approach to clinical decision making for psoriasis patients who fail anti-TNF therapy.
\end{abstract}

Keywords Psoriasis - Biologics · Anti-TNF . Immunogenicity $\cdot$ Anti-drug antibodies (ADA) $\cdot$ Drug trough levels

\section{Introduction}

Biologic agents such as the tumor necrosis factor alpha (TNF) antagonists etanercept, adalimumab and infliximab, as well as the interleukin (IL)-12/IL-23 antagonist ustekinumab, are available for the treatment of moderate to severe plaque psoriasis [9]. Although these biologics have proven to be highly efficacious, a significant number of patients will discontinue their first biologic because of primary failure (lack of initial efficacy), secondary failure

S. Bracke $(\bowtie) \cdot J$. Lambert

Department of Dermatology, Ghent University Hospital,

De Pintelaan 185, 9000 Ghent, Belgium

e-mail: stefanie.bracke@uzgent.be (loss of efficacy with time) or because of adverse reactions [16]. For clinicians considering the treatment options for a patient who has failed one TNF antagonist, it remains a challenge to determine the optimal next therapeutic step. In current clinical practice, drug switching is carried out without the knowledge of factors that may explain the failure.

An association between the occurrence of antidrug antibodies (ADAs), diminished trough serum drug levels (TSDLs) and non-response or loss of response has been described for several biologics in a variety of diseases, including psoriasis [3, 14, 25]. Nevertheless, studies investigating immunogenicity in psoriasis are scarce, and most of our current knowledge comes from studies in patients with rheumatoid arthritis (RA) or Crohn's disease [14]. By presenting ten cases of psoriasis patients with primary or secondary failure, or adverse reactions to a TNF antagonist and reviewing relevant articles, we want to show the clinical relevance of measuring TSDLs and/or ADAs in psoriasis patients and how this can direct a clinician's decision making.

\section{Materials and methods}

Ten patients with primary or secondary failure, or adverse reactions to a TNF antagonist (infliximab, etanercept, adalimumab) were seen at the Department of Dermatology at the Ghent University Hospital. Treatment success was defined as achieving PASI 75 and treatment failure when PASI 50 was not achieved, according to the European consensus guidelines [22]. Serum was collected at drug trough level (just before next administration of the drug), and the samples were analyzed at The Laboratory for Monoclonal Therapeutics, Sanquin Diagnostics, Amsterdam (the 
Netherlands). ADAs were detected by radioimmunoassay (RIA) and TSDLs were measured by enzyme linked immunosorbent assay (ELISA). The antibody test was considered positive when antibody concentrations exceeded $12 \mathrm{AE} / \mathrm{ml}$. Therapeutic TSDLs are: adalimumab $\geq 5 \mu \mathrm{g} / \mathrm{ml}$, infliximab $\geq 1 \mu \mathrm{g} / \mathrm{ml}$ and etanercept $\geq 2 \mu \mathrm{g} / \mathrm{ml}$ (values provided by Sanquin Diagnostics, Amsterdam, the Netherlands).

\section{Results}

Primary failure

Patient 1 did not respond to treatment with adalimumab and had a pronounced psoriasis exacerbation after 20 weeks. Anti-adalimumab antibodies were negative, and TSDLs were adequate $(13 \mu \mathrm{g} / \mathrm{ml})$. We continued treatment and after 24 weeks PASI 50 was achieved, and PASI 100 after 35 weeks.

Patient 2 had not achieved PASI 75 after 16 weeks of treatment with adalimumab. Testing showed no ADAs and adequate TSDLs $(7 \mu \mathrm{g} / \mathrm{ml})$. Finally, after 21 weeks of treatment, PASI 75 was achieved. Unfortunately, 8 weeks later, psoriasis relapsed. With addition of methotrexate (MTX) $7.5 \mathrm{mg} /$ week, PASI 75 was achieved after 2 months. The patient is currently still in remission (1 year and 9 months later).

Secondary failure

Patient 3 received adalimumab treatment for 1 year, stopped treatment for 6 weeks, and was subsequently nonresponsive to treatment for the following 7 months. Antiadalimumab antibodies $(800 \mathrm{AE} / \mathrm{ml})$ were present in the patient's serum at this point in time, yet TSDLs were undetectable as treatment had already been interrupted. Therapy was subsequently switched to ustekinumab, and PASI 100 was achieved after 12 weeks.

Patient 4 rapidly responded to adalimumab, but became non-responsive after 9 months. Temporary addition of MTX $5 \mathrm{mg} /$ week (3 months) had no effect. Anti-adalimumab antibodies were detected $(18 \mathrm{AE} / \mathrm{ml})$, but TSDLs were within therapeutic range $(14 \mu \mathrm{g} / \mathrm{ml})$. Therapy was switched to infliximab, but treatment was prematurely discontinued (see adverse reactions). Good clinical response was achieved by switching to ustekinumab.

Patient 5 achieved a PASI 75 during treatment with infliximab and MTX (5 mg/week), but became nonresponsive after 7 months. TSDLs were subtherapeutic, but no ADAs were detected. A good clinical response was achieved using ustekinumab. After 2 years treatment with infliximab, Patient 6 also became non-responsive but no
ADAs were detected and TSDLs were therapeutic $(17 \mu \mathrm{g} /$ $\mathrm{ml}$ ). Addition of MTX (5 mg/week) was sufficient to achieve disease control.

Patient 7 was successfully treated with etancercept for 2 years, after which psoriasis relapsed. Switching to adalimumab restored clinical response, but after 4 months psoriasis exacerbated again. Anti-adalimumab antibodies were positive $(24 \mathrm{AE} / \mathrm{ml})$. Readministration of etanercept for 18 months was unsuccessful. No anti-etanercept antibodies were detected, but TSDLs were subtherapeutic $(0.3 \mu \mathrm{g} / \mathrm{ml})$. Dose escalation $(2 \times 50 \mathrm{mg} / \mathrm{w})$ did not improve efficacy. By switching to ustekinumab, PASI 75 was finally achieved.

Patient 8 became non-response to infliximab therapy after 1 year. Anti-infliximab antibodies were positive (2,900 AE/ml). Psoriasis improved by switching to etanercept, but the patient became non-responsive after 1.5 years of treatment. No anti-etanercept antibodies were detected. Subsequent treatment with ustekinumab was successful.

\section{Adverse reactions}

Patient 4 developed nausea, flushing, itching during infliximab infusion and treatment was subsequently terminated. No anti-infliximab antibodies were detected (1 year later).

Patient 9 developed a skin rash after 4 weeks etanercept treatment. This skin rash was pathologically scored as a vacuolopathic parakeratotic interphase dermatitis, indicating a toxic reaction to medication. No anti-etanercept antibodies were detected. Good clinical response was achieved with ustekinumab.

Patient 10 developed erythroderma during etanercept treatment. No anti-etanercept antibodies were detected. Therapy was switched to infliximab, but after two successful treatment years, psoriasis relapsed with the development of anti-infliximab antibodies $(230 \mathrm{AE} / \mathrm{ml})$. Switching to ustekinumab gave good disease control.

\section{Discussion}

In the 2 patients we describe with primary failure (patient 1 and 2), testing showed adequate TSDLs and no ADAs. Findings in RA patients suggest that the absence of ADAs in non-responders might reflect a lack of responsiveness to the mechanism of action shared by all anti-TNF agents and indicates the need to switch to a drug with a different mechanism of action [3,11]. Accordingly, in patient 1 and 2, a switch to ustekinumab would be recommended (see Fig. 1a). Although some authors have showed the effectiveness and tolerability of switching between biologics in 


\section{a Primary or secondary failure to adalimumab or infliximab}

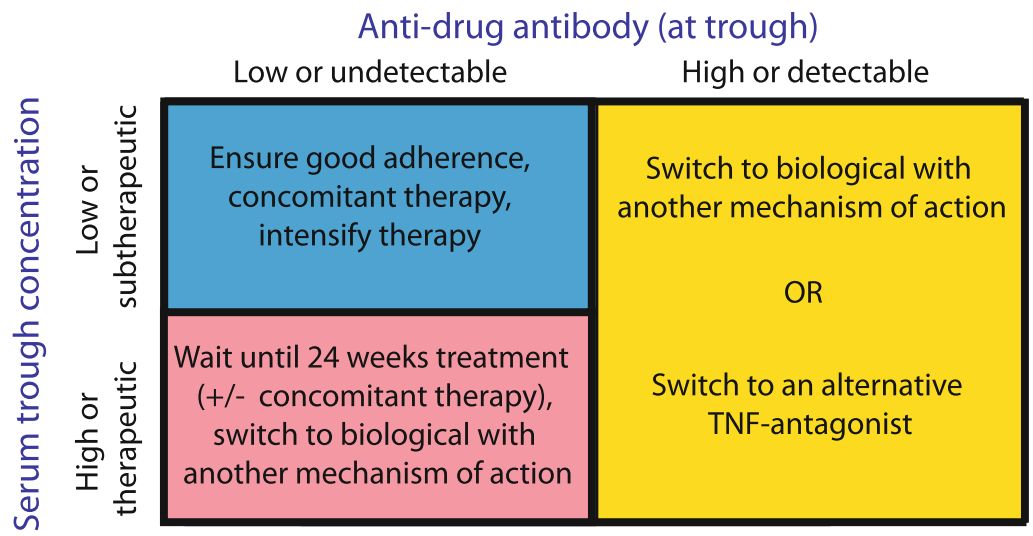

\section{b Primary or secondary failure to etanercept}

\section{Serum trough concentration}

High or therapeutic

Low or subtherapeutic

Wait until 24 weeks treatment (+/- concomitant therapy), switch to biological with another mechanism of action

Ensure good adherence, concomitant therapy, intensify therapy

\section{c Adverse reaction to adalimumab, infliximab or etanercept}

Fig. 1 Proposed treatment algorithm for psoriasis patients with primary or secondary failure, or adverse reactions to TNF antagonists a Primary or secondary failure to adalimumab or infliximab. Combining the results of trough serum drug levels (TSDLs) and anti-drug antibodies (ADAs) results in the following options: if the TSDLs are low, and no ADAs are detected, it is firstly important to ensure a good adherence. Concomitant treatment or intensification of treatment (increasing dosage or decreasing treatment interval) can be considered. In the case of therapeutic TSDLs without ADA formation, we recommend to wait until 24 weeks of treatment has passed before switching to a biologic with another mechanism of action. Concomitant therapy may be considered. When ADAs are present, both switching to an alternative TNF antagonist or a biologic with another mechanism of action are good options. b Primary or secondary failure to etanercept. In patients treated with etanercept, ADAs are reported to be non-neutralizing, and therefore only TSDLs are considered. If TSDLs are within the therapeutic range, we recommend switching to a biologic with another mechanism of action after a treatment period of 24 weeks. Concomitant therapy may be considered. In the case of low or subtherapeutic TSDLs, intensification of treatment, ensuring a good adherence, and/or concomitant therapy may enhance efficacy. c Adverse reaction to adalimumab, infliximab or etanercept. In the case of adverse reactions, the value of ADA or TSDL testing is questionable. Both a switch to an alternative TNF antagonist and a biologic with another mechanism of action are good options 
psoriasis patients [13], it is not recommended to switch too often as intermittent treatment or re-exposure after a treatment free interval may be associated with an increase in immunogenicity $[1,15]$. The National Institute for Health and Clinical Excellence (NICE) recommends evaluating the treatment success of biologics at 10 weeks for infliximab, 12 weeks for etanercept and 16 weeks for both adalimumab and ustekinumab [24]. However, in several studies, even more patients achieved PASI 75 after 24 weeks [17, 18, 21, 23]. Therefore, we recommend waiting at least 24 weeks before concluding the treatment is not working, particularly when ADAs are not detected, to avoid potentially unnecessary switching. Concomitant treatment may be considered as this may enhance efficacy [24] (Fig. 1a, b). Accordingly, as in patient 1 and 2 no ADAs were detected and TSDLs were adequate, we extended treatment and no switch was required as both patients achieved PASI 75 at 24 weeks.

In patients 3 and 4, secondary failure was associated with anti-adalimumab antibodies. Based on the literature, a switch to another TNF antagonist can be efficacious in such cases, as ADAs do not crossreact [3, 11, 16] (Fig. 1a). Unfortunately, patient 4 developed an infusion reaction during the first administration of infliximab, and therapy was switched to ustekinumab with good response.

Patient 5 became unresponsive to infliximab treatment. No ADAs were detected, but infliximab serum levels were subtherapeutic. In patients with subtherapeutic concentrations, infliximab dose escalation (i.e. increasing the dose or shortening of the administration interval between infusions) has been associated with a significantly increased clinical response compared to changing to another antiTNF [2]. Another study also reported dose escalation to be a good choice in patients negative for ADAs with low serum infliximab levels [26]. Unfortunately, due to governmental reimbursement restrictions, dose escalation could not be performed in our patient. Patient 6 also became unresponsive to infliximab. No ADAs were detected, and TSDLs were therapeutic. Detectable infliximab trough concentrations have been associated with better clinical remission rates [19], but as discussed earlier; addition of concomitant treatments may enhance efficacy $[8,24]$. Therefore, we decided to maintain the treatment dosing schedule in our patient, but additionally prescribed MTX (Fig. 1a) which resulted in an improved treatment efficacy.

In patient 7, readministration of etanercept therapy after adalimumab failure was not successful. No ADAs were detected, but TSDLs were subtherapeutic. Potential explanations for low TSDLs without ADA formation include patient non-compliance, or an inadequate drug dosage for the degree of inflammation as has been described for infliximab $[7,26]$. Thus, patient 7 was treated with
Table 1 Trough serum drug levels and anti-drug antibody levels of 10 psoriasis patients

\begin{tabular}{lllll}
\hline Patient & $\begin{array}{l}\text { Biologic } \\
\text { agent }\end{array}$ & $\begin{array}{l}\text { TSDLs }^{\mathrm{a}} \\
(\mu \mathrm{g} / \mathrm{ml})\end{array}$ & $\begin{array}{l}\mathrm{ADAs}^{\mathrm{b}} \mathrm{IgG} \\
\text { totaal }(\mathrm{AE} / \mathrm{ml})\end{array}$ & $\begin{array}{l}\text { Primary } \\
(\mathrm{P}) \text { or } \\
\text { secondary } \\
\text { (S) failure, } \\
\text { or adverse } \\
\text { (A) reaction }\end{array}$ \\
\hline 1 & Adalimumab & 13 & $<12$ & $\mathrm{P}$ \\
2 & Adalimumab & 7 & $<12$ & $\mathrm{P}$ \\
3 & Adalimumab & $<0.002$ & 800 & $\mathrm{~S}$ \\
4 & Adalimumab & 14 & 18 & $\mathrm{~S}$ \\
& Infliximab & $N / A$ & $<12($ after 1 year) & $\mathrm{A}$ \\
5 & Infliximab & 0.7 & $<12$ & $\mathrm{~S}$ \\
6 & Infliximab & 17 & $<12$ & $\mathrm{~S}$ \\
7 & Adalimumab & $<0.002$ & 24 & $\mathrm{~S}$ \\
& Etanercept & 0.3 & $<12$ & $\mathrm{~S}$ \\
8 & Infliximab & $<0.002$ & 2900 & $\mathrm{~S}$ \\
& Etanercept & 3 & $<12$ & $\mathrm{~S}$ \\
9 & Etanercept & $<0.007$ & $<12$ & $\mathrm{~A}$ \\
10 & Etanercept & $<0.002$ & $<12$ & $\mathrm{~A}$ \\
& Infliximab & $<0.002$ & 230 & $\mathrm{~S}$ \\
\hline
\end{tabular}

Italic blood drawn after discontinuation of treatment

a Therapeutic TSDLs adalimumab $\geq 5 \mu \mathrm{g} / \mathrm{ml}$, infliximab $\geq 1 \mu \mathrm{g} / \mathrm{ml}$, etanercept $\geq 2 \mu \mathrm{g} / \mathrm{ml}$ (values provided by Sanquin Diagnostics, Amsterdam, the Netherlands)

b ADAs positive $>12 \mathrm{AE} / \mathrm{ml}$

an increased dose of etanercept. Unfortunately, the patient remained non-responsive. Similarly, in patient 8 , switching to etanercept after previous TNF-antagonist failure was not successful. Although etanercept may represent an effective and well-tolerated alternative treatment for psoriasis patients who have failed to respond to other therapies (traditional or biologic), it has been reported that etanercept was more effective in biologic naive patients [20]. However, the rate of response to a second TNF antagonist is usually inferior to that of the first [16]. On the other hand, patients who discontinued their first anti-TNF agent due to adverse effects are more likely to respond to another TNF antagonist than those who experienced inefficacy [10]. Adverse reactions were observed in patients 9 and 10 during treatment with etanercept. Patient 10 indeed responded well to a switch to infliximab. However, after 2 years, ADAs against infliximab developed and therapy was switched to ustekinumab. In patient 9 , therapy was immediately switched to ustekinumab. Patient 4 developed an acute infusion reaction to infliximab. No anti-infliximab antibodies were detected (1 year later). Although anti-infliximab antibodies may favor the occurrence of infusion reactions, their presence is not specific [4].

From a clinical and economic perspective, testing of ADAs and TSDLs may not be worthwhile in the case of 
adverse reactions as switching is the only option in the case of severe adverse reactions [15]. In such a setting, different TNF-antagonists or biologics with different mechanisms of action are viable treatment alternatives (Fig. 1c).

In none of the patients treated with etanercept (patients 7, 8, 9 and 10), ADAs against the drug were detected. In general, anti-etanercept antibodies have only been reported scarcely [6]. However, two studies in RA patients showed that non-responders to etanercept obtain lower serum drug levels compared to responding patients $[5,12]$. Therefore, in etancercept-treated patients, we suggest to only consider testing of TSDLs. In the case of subtherapeutic etanercept trough levels, we propose the etanercept dosage to be increased. In the case of therapeutic trough levels, a switch to another class is recommended (Fig. 1b).

Despite some methodological shortcomings with respect to blood samples drawn from patients post-treatment (see Table 1), we show that measuring ADAs and/or TSDLs can guide a dermatologist's decision-making process in a more pragmatic way. Looking at TNF antagonists from a pharmacological point of view (measuring therapeutic trough levels and titrate dose accordingly) is rather new, and could in the future help us to dose these drugs in a more cost effective manner (e.g., in case of good response and high trough levels one could lower the dose of drug).

In conclusion, we show on a case-by-case basis that measuring ADAs and TSDLs can optimise biologic treatment in psoriasis patients. Our report provides a first step towards a more structured approach when dealing with non-response or loss of response to biologics. Our preliminary treatment algorithm provides a new viewpoint, which encompasses the currently available literature. Further research is needed to clarify its applicability to improve the outcomes of patients treated with TNF antagonists.

Acknowledgments Dr. S. Bracke is funded by an IWT Grant (091208) ('Flemish government agency for Innovation by Science and Technology').

Conflict of interest None.

\section{References}

1. (EMEA) EAftEoMP (2007) Guideline on immunogenicity assessment of biotechnology-derived therapeutic proteins. EMEA/CHMP/BMWP/14327/2006

2. Afif W, Loftus EV Jr, Faubion WA, Kane SV, Bruining DH, Hanson KA, Sandborn WJ (2010) Clinical utility of measuring infliximab and human anti-chimeric antibody concentrations in patients with inflammatory bowel disease. Am J Gastroenterol 105(5):1133-1139

3. Bartelds GM, Wijbrandts CA, Nurmohamed MT, Stapel S, Lems WF, Aarden L, Dijkmans BA, Tak PP, Wolbink GJ (2010) Anti- infliximab and anti-adalimumab antibodies in relation to response to adalimumab in infliximab switchers and anti-tumour necrosis factor naive patients: a cohort study. Ann Rheum Dis 69(5):817-821

4. Chavez-Lopez MA, Delgado-Villafana J, Gallaga A, HuertaYanez G (2005) Severe anaphylactic reaction during the second infusion of infliximab in a patient with psoriatic arthritis. Allergol Immunopathol (Madr) 33(5):291-292

5. Daien CI, Daien V, Parussini E, Dupuy AM, Combe B, Morel J (2012) Etanercept concentration in patients with rheumatoid arthritis and its potential influence on treatment decisions: a pilot study. J Rheumatol 39(8):1533-1538

6. Dore RK, Mathews S, Schechtman J, Surbeck W, Mandel D, Patel A, Zhou L, Peloso P (2007) The immunogenicity, safety, and efficacy of etanercept liquid administered once weekly in patients with rheumatoid arthritis. Clin Exp Rheumatol 25(1):40-46

7. Edrees AF, Misra SN, Abdou NI (2005) Anti-tumor necrosis factor (TNF) therapy in rheumatoid arthritis: correlation of TNFalpha serum level with clinical response and benefit from changing dose or frequency of infliximab infusions. Clin Exp Rheumatol 23(4):469-474

8. Guenther LC (2011) Combination therapy of biologics with traditional agents in psoriasis. Skin Therapy Lett 16(6):1-3

9. Herrier RN (2011) Advances in the treatment of moderate-tosevere plaque psoriasis. Am J Health Syst Pharm 68(9):795-806

10. Hyrich KL, Lunt M, Watson KD, Symmons DP, Silman AJ, British Society for Rheumatology Biologics R (2007) Outcomes after switching from one anti-tumor necrosis factor alpha agent to a second anti-tumor necrosis factor alpha agent in patients with rheumatoid arthritis: results from a large UK national cohort study. Arthritis Rheum 56(1):13-20

11. Jamnitski A, Bartelds GM, Nurmohamed MT, van Schouwenburg PA, van Schaardenburg D, Stapel SO, Dijkmans BA, Aarden L, Wolbink GJ (2011) The presence or absence of antibodies to infliximab or adalimumab determines the outcome of switching to etanercept. Ann Rheum Dis 70(2):284-288

12. Jamnitski A, Krieckaert CL, Nurmohamed MT, Hart MH, Dijkmans BA, Aarden L, Voskuyl AE, Wolbink GJ (2012) Patients non-responding to etanercept obtain lower etanercept concentrations compared with responding patients. Ann Rheum Dis 71(1):88-91

13. Lecluse LL, de Groot M, Bos JD, Spuls PI (2009) Experience with biologics for psoriasis in daily practice: switching is worth a try. Br J Dermatol 161(4):948-951

14. Lecluse LL, Driessen RJ, Spuls PI, de Jong EM, Stapel SO, van Doorn MB, Bos JD, Wolbink GJ (2010) Extent and clinical consequences of antibody formation against adalimumab in patients with plaque psoriasis. Arch Dermatol 146(2):127-132

15. Lecluse LL, Piskin G, Mekkes JR, Bos JD, de Rie MA (2008) Review and expert opinion on prevention and treatment of infliximab-related infusion reactions. Br J Dermatol 159(3):527-536

16. Leman J, Burden AD (2012) Sequential use of biologics in the treatment of moderate-to-severe plaque psoriasis. Br J Dermatol 167(Suppl 3):12-20

17. Leonardi CL, Kimball AB, Papp KA, Yeilding N, Guzzo C, Wang Y, Li S, Dooley LT, Gordon KB, investigators Ps (2008) Efficacy and safety of ustekinumab, a human interleukin-12/23 monoclonal antibody, in patients with psoriasis: 76-week results from a randomised, double-blind, placebo-controlled trial (PHOENIX 1). Lancet 371(9625):1665-1674

18. Leonardi CL, Powers JL, Matheson RT, Goffe BS, Zitnik R, Wang A, Gottlieb AB, Etanercept Psoriasis Study G (2003) Etanercept as monotherapy in patients with psoriasis. N Engl $\mathrm{J}$ Med 349(21):2014-2022

19. Maser EA, Villela R, Silverberg MS, Greenberg GR (2006) Association of trough serum infliximab to clinical outcome after 
scheduled maintenance treatment for Crohn's disease. Clin Gastroenterol Hepatol 4(10):1248-1254

20. Mazzotta A, Esposito M, Costanzo A, Chimenti S (2009) Efficacy and safety of etanercept in psoriasis after switching from other treatments: an observational study. Am J Clin Dermatol 10(5):319-324

21. Mease PJ, Ory P, Sharp JT, Ritchlin CT, Van den Bosch F, Wellborne F, Birbara C, Thomson GT, Perdok RJ, Medich J, Wong RL, Gladman DD (2009) Adalimumab for long-term treatment of psoriatic arthritis: 2-year data from the Adalimumab Effectiveness in Psoriatic Arthritis Trial (ADEPT). Ann Rheum Dis 68(5):702-709

22. Mrowietz U, Kragballe K, Reich K, Spuls P, Griffiths CE, Nast A, Franke J, Antoniou C, Arenberger P, Balieva F, Bylaite M, Correia O, Dauden E, Gisondi P, Iversen L, Kemeny L, Lahfa M, Nijsten T, Rantanen T, Reich A, Rosenbach T, Segaert S, Smith C, Talme T, Volc-Platzer B, Yawalkar N (2011) Definition of treatment goals for moderate to severe psoriasis: a European consensus. Arch Dermatol Res 303(1):1-10
23. Reich K, Nestle FO, Papp K, Ortonne JP, Evans R, Guzzo C, Li S, Dooley LT, Griffiths CE (2005) Infliximab induction and maintenance therapy for moderate-to-severe psoriasis: a phase III, multicentre, double-blind trial. Lancet 366(9494):1367-1374

24. Smith CH, Anstey AV, Barker JN, Burden AD, Chalmers RJ, Chandler DA, Finlay AY, Griffiths CE, Jackson K, McHugh NJ, McKenna KE, Reynolds NJ (2009) Ormerod AD (2009) British Association of Dermatologists' guidelines for biologic interventions for psoriasis. Br J Dermatol 161(5):987-1019

25. Spuls PI, Lecluse LL, Poulsen ML, Bos JD, Stern RS, Nijsten T (2010) How good are clinical severity and outcome measures for psoriasis?: quantitative evaluation in a systematic review. J Invest Dermatol 130(4):933-943

26. Takeuchi T, Miyasaka N, Tatsuki Y, Yano T, Yoshinari T, Abe T, Koike T (2011) Baseline tumour necrosis factor alpha levels predict the necessity for dose escalation of infliximab therapy in patients with rheumatoid arthritis. Ann Rheum Dis 70(7): $1208-1215$ 